\title{
Bellum Iustum - Eine moraltheologische Auseinandersetzung bei Karl Hörmann
}

\author{
Birgit Rath
}

This paper will give an insight into the theological war discourse of the $20^{\text {th }}$ century. Focusing especially on the time after the Second World War, Karl Hörmann's scientific discourse (1915-2004) will be the pivotal element of this article. One of his main research areas was the bellum-iustum doctrine, which he restricted in a way that questioned the legitimacy of war, even though he also argued strictly against an imperative war ban. Hörmann's writings show a dichotomy, which in a certain way reflects a paradigmatic dilemma of his time. On the one hand, it demonstrates adherence to the traditional doctrines of just war and the right to defense. On the other hand, attempts of a new peace ethics become visible. Hörmann's teachings were marked by the obligation to defend justice and, at the same time, by a desire for peace.

bellum iustum, World War II, Second Vatican Council, peace ethics, nuclear war, Karl Hörmann

Birgit Rath is an university assistant (prae doc) at the Department of Systematic Theology and Ethics/ Theological Ethics at the University of Vienna. Her research activities focus on the history of moral theology. Recent publications: Müller, Sigrid / Rath, Birgit: "Sterben und Todesfeststellungen in der Medizingeschichte", in: Körtner, Ulrich / Kopetzki, Christian u.a. (Hrsg.), Hirntod und Organtransplantation - zum Stand der Diskussion, (Schriftenreihe Ethik und Recht in der Medizin; Band 12) Wien, 2016, S. 1-17. Müller, Sigrid / Novak, Melanie / Rath, Birgit: "Kidnapping (Christianity)", in: Klauck, Hans-Josepf / McGinn, Bernhard u.a. (Hrsg.): Encyclopedia of the Bible and Its Reception (EBR). (in print)

\section{Einleitung}

Seit der Antike galt die christlich-theologische Reflexion über den Krieg der Frage, unter welchen Bedingungen ein sogenannter bellum iustum - ein gerechter Krieg - möglich sei. So wurde die bellum-iustum-Lehre ${ }^{1}$ entwickelt, weiter aus-

1 „Damit ein K. gerecht ist, muss er aus einem gerechten Grund („,causa iusta“), mit dem Willen zum Frieden (,intentio recta“) und auf Befehl der rechten Autorität (,legitima auctoritas“) geführt werden. Wenn sich Christen an einem gerechten Krieg beteiligen, befolgen sie Jesu Liebesgebot. Thomas von Aquin bringt die drei Kriterien für einen gerechten K., die sich bei Augustinus verstreut finden, in die von da an übliche 
Birgit Rath

differenziert und auf der Basis von Augustinus und Thomas von Aquin bis ins frühe 20. Jahrhundert ohne größere Veränderungen tradiert, obwohl sich die Konditionen der Kriegsführung erheblich veränderten.

In dieser moraltheologischen Lehre wurde Krieg nicht grundsätzlich als unerlaubt angesehen, solange bestimmte Voraussetzungen innerhalb der Kriegsführung eingehalten wurden. Dementsprechend wurde die bellum-iustum-Lehre im 20. Jahrhundert eher als Rahmen betrachtet, welcher durch verschiedene Interpretationen, wie etwa durch den österreichischen Moraltheologen Karl Hörmann (1915-2004), eine Ausformung erfuhr, die auch eine grundsätzliche Infragestellung von Krieg gedanklich in Sichtweite brachte. ${ }^{2}$

Für Hörmann etwa stellte sich die Frage, ob ein Krieg in Bezug auf den einzelnen Menschen sittlich oder unsittlich sei, erst seit der Möglichkeit eines totalen Krieges - Hörmann meint einen Krieg, der alle gesellschaftlichen Ressourcen umfasst, insbesondere die industrielle Kriegsführung - sowie unter der Bedingung einer allgemeinen Wehrpflicht ${ }^{3}$. Zuvor war es zumindest theoretisch möglich, die Kriegsfrage für sich persönlich zu lösen, indem man dem Krieg fernblieb. ${ }^{4}$ Ein gewisses Umdenken innerhalb des theologischen Diskurses kann auch auf die beiden Weltkriege im zwanzigsten Jahrhundert und die Gräuel, die diese mit sich brachten, zurückgeführt werden. Ein wichtiger kirchlicher Vertreter innerhalb der bellum-iustum-Debatte des 20. Jahrhunderts war Pius XII. (1876-1958), dessen Lehren bis weit hin in die Amtszeit Johannes XXIII. (1881-1963) prägend für die moraltheologischen Auseinandersetzungen mit dem Krieg waren und die

Reihenfolge: legitime Autorität, gerechter Grund und rechte Intention (S. th. II-II, 40,1). “ Beestermöller, Gerhard, Krieg, in: Hunold, Gerfried W. / Sautermeister, Jochen (Hrsg.), Lexikon der christlichen Ethik Bd. 1, Herder, Freiburg / Basel / Wien, ${ }^{32003,}$ S. 1015.

2 Vgl. Nagel, Ernst-Josef / Oberhem, Harald, Dem Frieden verpflichtet. Konzeptionen und Entwicklungen der katholischen Friedensethik seit dem Zweiten Weltkrieg, Kaiser, München; Matthias-Grünewald, Mainz, 1982, S. 11-12.

3 „Unter diesem Gesichtspunkt hat die Kirche für Friedenszeiten Berufsheere den Heeren mit allgemeiner Wehrpflicht vorgezogen, weil sie den Staatsbürgern ein Mindestmaß an Lasten zumuten. Die allgemeine Wehrpflicht wurde erst seit Napoleons Zeiten in immer mehr Ländern eingeführt. Die Folgen waren ein Wettrüsten zwischen den Staaten, hohe Steuern für die Erhaltung der zahlenmäßig starken Heere und für immer neue Rüstungen, Berufseinschränkungen der Bürger, schließlich Kriege, die auch für die Sieger nicht immer mit wirklichem Gewinn endeten. "Hörmann, Karl, Friede und moderner Krieg im Urteil der Kirche, Wiener Dom-Verlag, Wien, 1964, S. 67. Nach Hörmann haben sich bereits Leo XIII. (1810-1903) und Benedikt XV. (1854-1922) gegen die allgemeine Wehrpflicht ausgesprochen, letzterer bezeichnete deren Abschaffung sogar als Voraussetzung für den Frieden, da sie der Grund für unzählige Übel sei. Vgl. K. Hörmann, Friede und moderner Krieg, S. 67.

4 Vgl. Hörmann, Karl, Lexikon der christlichen Moral, Tyrolia, Innsbruck / Wien / München, 1969, S. 705. 
auch in Hörmanns Schriften Widerhall finden. Pius XII. versuchte seine Lehre als Gegenpol zu Kommunismus und Nationalismus in Richtung einer Friedenstheorie auszulegen. ${ }^{5}$

Im folgenden Artikel soll nun exemplarisch einem Ausschnitt innerhalb des theologischen Kriegsdiskurses Mitte des 20. Jahrhunderts anhand der wissenschaftlichen Auseinandersetzung Karl Hörmanns mit diesem Thema nachgegangen werden. Hörmanns Lehrtätigkeit begann 1953 am Institut für Moraltheologie an der Katholisch-Theologischen Fakultät in Wien, wo er zunächst als außerordentlicher Professor arbeitete und 1959 zum ordentlichen Professor für Moraltheologie ernannt wurde. An der Universität Wien fungierte er unter anderem als Senator der Universität, war 1963/64 und 1973/74 Dekan der Katholisch-Theologischen Fakultät und bekleidete 1966/67 das Amt des Rektors der Universität Wien, bevor er 1985 emeritierte. ${ }^{6}$ Während dieser Zeit arbeitete er darüber hinaus in der Laienapostolischen Bewegung und auf diversen internationalen Kongressen als Beobachter für den Vatikan. Geprägt war seine Lehrtätigkeit besonders durch den Zweiten Weltkrieg und seine Folgen. Die Thematik des Krieges spiegelt sich in zahlreichen wissenschaftlichen Publikationen und Vorträgen Hörmanns wider ${ }^{7}$ und ist gleichsam ein Abbild des damals vorherrschenden Zeitgeistes. So gab es sowohl innerhalb der Völkerrechtsdiskussion eine intensive Auseinandersetzung mit der bellum-iustum-Lehre, als auch innerhalb des moralphilosophischen Diskurses. ${ }^{8}$

5 Vgl. Nagel, Ernst-Josef / Oberhem, Harald, Dem Frieden verpflichtet, S. 12, S. 32.

6 Vgl. Virt, Günter (Hrsg.), Spiritualität in Moral. Festschrift für Karl Hörmann zum 60. Geburtstag, Wiener Dom-Verlag, Wien, 1975, S. IX; Vgl. Virt, Günter, Karl Hörmann, in: Bautz, Friedrich Wilhelm / Bautz, Traugott (Hrsg.), Biographisch-Bibliographisches Kirchenlexikon Bd. 27, Ergänzungen XIV, Traugott Bautz, Nordhausen, 2007, S. 674. Vgl. Kathpedia: Karl Hörmann, online abrufbar unter: http://www.kathpedia. com/index.php?title=Karl_H\% C3\% B6rmann [09.02.2017].

7 Vgl. Hörmann, Karl, Sittliche Probleme des modernen Krieges, in: Religion Wissenschaft - Kultur 8 (1957) S. 221-226; Eid, Befehl, Widerstand, Tyrolia, Innsbruck / Wien / München, 1964; Der Wert sittlicher Überlegungen über den Krieg. Inaugurationsrede gehalten am 20. Oktober 1966, Wien, 1966; Wehrdienst - Kriegsdienst - Verweigerung - gerechter Krieg?, Winfried-Werk, Augsburg, 1971; Der „gerechte Krieg“ im christlichen Denken, in: Weiler, Rudolf / Zsifkovits, Valentin (Hrsg.), Unterwegs zum Frieden, Herder, Freiburg / Basel / Wien, 1973, S. 335-367.

8 Vgl. Jensen, Jessica, Krieg um des Friedens willen. Zur Lehre vom gerechten Krieg, Nomas, Baden-Baden, 2015, S. 283. Hintergrund für diese Beschäftigung bilden unter anderem auch die Friedensbewegungen der 1960er und 70er Jahre. An dieser Stelle seien etwa die ökumenischen Friedensbewegungen und deren sozialethische Auseinandersetzungen mit den Befreiungskämpfen der Völker der „Dritten Welt“ zu nennen. Diese Befreiungsbewegungen, der aktive Widerstand bei Menschenrechtsverletzungen und der Kampf gegen die kolonialen und postkolonialen Strukturen wurden weitgehend als „gerechter Krieg“ angesehen. Vgl. J. Jensen, Krieg um des Friedens willen, S. 283-284. 
Birgit Rath

In seinem Standardwerk „Lexikon der christlichen Moral“ von 1969 definiert Karl Hörmann den Krieg als gewaltsame Austragung von Streitigkeiten durch Staaten oder staatlich organisierte Großgruppen, welche von der Sittlichkeitslehre, die an der Offenbarung orientiert ist, nie als etwas Wünschenswertes betrachtet werde. Der Krieg widerspricht demnach dem Erfahrungswissen über die Übel, welche er hervorruft, außerdem dem Auftrag zum Frieden als Teil der Offenbarung und ebenso der Erkenntnis, wonach Gewalt nicht der menschenwürdige Weg zur Durchsetzung von Recht sei oder diesem sogar entgegenstehe. In seiner Argumentation verweist Hörmann auf die Ablehnung des Krieges, wie sie auf dem Zweiten Vatikanischen Konzil (1962-1965) in der Pastoralkonstitution „Gaudium et Spes“ (GS) in Kapitel V ${ }^{9}$ festgehalten wurde. Auf diesem wurden die bellum-iustum-Lehre auf die Selbstverteidigung eingeschränkt und jegliche Strategien, welche auf pure Zerstörung zielten, strikt abgelehnt.

Doch trotz dieser ablehnenden Haltung stellt es für Hörmann keine Option dar, den Krieg und die Anwendung von Gewalt gänzlich zu verbieten. Es müsse grundsätzlich erlaubt bleiben, sich im äußersten Fall zu verteidigen, einschließlich kriegerischer Mittel. ${ }^{10}$ Diesbezüglich argumentiert er zwar, dass ein Krieg nur als

Dennoch wurden auch Zweifel daran geäußert, ob Gewaltanwendung jemals zu einer Verbesserung einer Situation führen könne, wie etwa von Paul VI. in Populorum Progessio 31. „Jede Revolution - ausgenommen im Fall der eindeutigen und lange dauernden Gewaltherrschaft, die die Grundrechte der Person schwer verletzt und dem Gemeinwohl des Landes ernsten Schaden zufügt - zeugt neues Unrecht, bringt neue Störungen des Gleichgewichts mit sich, ruft neue Zerrüttung hervor. Man kann das Übel, das existiert, nicht mit einem noch größeren Übel vertreiben.“ http://w2.vatican.va/con tent/paul-vi/de/encyclicals/documents/hf_p-vi_enc_26031967_populorum.html [07.02.2018].

9 „Es ist also deutlich, daß wir mit all unseren Kräften jene Zeit vorbereiten müssen, in der auf der Basis einer Übereinkunft zwischen allen Nationen jeglicher Krieg absolut geächtet werden kann.“ GS 82 http://www.vatican.va/archive/hist_councils/ii_vatican_ council/documents/vat-ii_const_19651207_gaudium-et-spes_ge.html [01.08.2017].

10 Auch diese Argumentation geschieht in Anlehnung an das Zweite Vatikanum. In GS 79 etwa wird das Recht auf sittlich erlaubte Verteidigung gebilligt. Jedoch knüpft Hörmann kriegerische Handlungen an bestimmte Bedingungen und nur unter deren Einhaltung kann ein Krieg als gerecht bezeichnet werden. Diese Bedingungen ergeben sich aus dem Notmittelcharakter des Krieges und aus sittlichen Überlegungen. Zunächst müsse es einen schweren und gerechten Grund geben, welcher sich jedoch kaum feststellen lasse, da Kriegsursachen meist sehr komplexe Zusammenhänge darstellen. Der einzige gerechte Grund, den Hörmann nennt, ist ein erlittenes Unrecht, dass ein Gegner nicht gutmachen will. Dies impliziert, dass zuerst Genugtuung gefordert werden müsse, die in jedem Fall anzunehmen sei. Eine weitere Bedingung stellt die Proportionalität dar. Die Gründe müssen entsprechend schwerer sein als die Übel, welche der Krieg mit sich bringt. Ferner müsse es eine Gewissheit geben, siegen zu können. „Aus Verantwortung gegenüber den eigenen Staatsbürgern darf die Führung eines Staates einen K. nicht unternehmen, wen sie wahrscheinl. Unterliegen wird, od. wenn selbst im Fall des Sieges 
Notmittel zulässig sein könne, da der Frieden dem christlichen Ideal entspräche zur Untermauerung seiner Argumente verweist er auf das Alte Testament, die christliche Liturgie und die Kirchenväter, ebenso auf Pius XII. und Theologen wie Karl Barth -, dennoch spricht er sich im Sinne der katholischen Lehre gegen ein grundsätzliches Verbot aus.

Demgemäß sind Hörmanns Schriften durch das Festhalten an einer traditionellen katholischen Argumentation geprägt, auch wenn er zugleich aufzuzeigen versucht, dass die Kriege des 20. Jahrhunderts mit keinen vorhergehenden vergleichbar sind. Deshalb lässt sich trotz aller Vorbehalte gegenüber einem „falschen Pazifismus "11, wie Hörmann ihn nennt, eine klare Ablehnung des Krieges ablesen. Hörmann selbst scheint demnach in einen Zwiespalt geraten zu sein, einerseits dem Krieg negativ gegenüberzustehen, gleichzeitig jedoch einem gewissen Rechtfertigungsdruck ${ }^{12} \mathrm{zu}$ unterliegen, die katholische Lehre verteidigen zu müssen. Für ihn stellt das gänzliche Ablehnen jeglicher Gewalt keine Option dar, da jedem Einzelnen das Recht auf Verteidigung zugestanden werden müsse

der Schaden wahrscheinl. Größer sein wird als der Nutzen. Dabei ist nicht nur das eigene Volk zu berücksichtigen, sondern auch die Gemeinschaft der Völker.“ K. Hörmann, Lexikon, S. 707. Als weitere Bedingung nennt Hörmann die rechte Absicht der Kriegsführenden, ausschließlich das eigene Recht schützen zu wollen. Ein Krieg dürfe darüber hinaus nicht ohne Kriegserklärung begonnen werden und nach Erlangen des Rechtes gäbe es eine Verpflichtung zu Verhandlungen. Im Falle eines Unterliegens sei man meistens dazu verpflichtet, das Friedensdiktat anzunehmen, um seinen Mitbürgern größeren Schaden zu ersparen. Damit ein gerechter Krieg gerecht bleibt, müsse er gerecht geführt werden, also unter der Einhaltung der natur- und völkerrechtlichen Richtlinien, wie bspw. ein Verbot der Tötung von Nichtkämpfern. Vgl. K. Hörmann, Lexikon, S. 706-708.

11 „Es gibt einen Typ des Pazifisten, bei dem wir bezweifeln müssen, ob er der Sache des Friedens einen wirklichen Dienst erweist: jenen, der selbstgefällig die Menschen früherer Zeiten und die Vertreter anderer Ansichten aburteilt; der geneigt ist, den Andersdenkenden die ernste Sorge um den Frieden abzusprechen und sie nur für sich in Anspruch zu nehmen; der nicht dessen gewahr wird, daß er selbst zu primitiv, vielleicht sogar falsch argumentiert; der einen falschen Frieden verficht und so tut, als ob das Leben der Güter höchstes wäre, dessen Einsatz auf keinen Fall gefordert werden dürfe, und den Krieg mehr wegen der damit verbundenen physischen Übel als wegen seiner Ungerechtigkeit verabscheut.“ K. Hörmann, Friede und moderner Krieg, S. 20. Diese Ablehnung des falschen Pazifismus betont Hörmann des Öfteren, auch in Anlehnung an andere Theologen, wie etwa Werner Schöllgen, welcher ebenfalls an dem Recht auf Verteidigung im Falle eines Angriffs festhält. Vgl. K. Hörmann, Friede und moderner Krieg, S. 42.

12 „Ist damit die Lehre der früheren Moraltheologen als unrichtig erkannt? Wenn es so wäre, würde dies nichts gegen die Lehre der Kirche sagen, denn die Kirche als solche deckt keine Kriegstheorie irgendwelcher Theologen der Vergangenheit; es gibt ja kein definiertes Dogma über den Krieg.“ K. Hörmann, Friede und moderner Krieg, S. 40. 
Birgit Rath

und insbesondere Staaten in der Pflicht stünden, ihr Volk zu verteidigen. ${ }^{13}$ Diese miteinander im Widerstreit stehenden Positionen Hörmanns sollen nun im Folgenden näher erläutert werden.

\section{Die Ablehnung eines Kriegsverbotes}

Hörmanns ethische Auseinandersetzungen zum Krieg orientieren sich durchgehend an der bellum-iustum-Lehre, so stellt er etwa die kriegerische Verteidigung bzw. die Anwendung von Gewalt im Verteidigungsfall nicht infrage. Dennoch vertritt er die Meinung, man könne diese Lehre nicht unmittelbar in die heutige Zeit übertragen. Frühere Theologen hätten sich zwar bemüht, die sittlichen Grundsätze auf die Gegebenheiten ihrer Zeit anzuwenden. Diese theologischen Entwürfe könnten jedoch nicht wie eine Schablone auf das 20. Jahrhundert umgelegt werden, da sie unter anderen Verhältnissen festgehalten wurden. Die konkreten Ausformungen seien demnach nicht zeitlos, sondern zeitbedingt, weshalb man heute $\mathrm{zu}$ einem anderen ethischen Urteil kommen müsse als in früheren Zeiten, „weil der Krieg ein anderer geworden ist“"14.

Für Hörmann ist es nach dem Zweiten Weltkrieg also nicht mehr möglich, einen moraltheologischen Diskurs über den Krieg mit den Vorlagen der Scholastik zu führen, da sich die Bedingungen, die damals für einen gerechten Krieg aufgestellt wurden, nicht bzw. fast nicht erfüllen ließen. Der Krieg habe demnach eine neue Gestalt angenommen, vor allem in Form neuer Waffengattungen, wie zum Beispiel der ABC-Waffen. Dies bedeutet für Hörmann jedoch keine Ablehnung früherer moraltheologischer Lehren, sondern lediglich eine radikale Abkehr und Ächtung des modernen Angriffskrieges, wie sie bereits 1944 von Pius XII. in seiner Weihnachtsansprache ausgesprochen und später in „Gaudium et

13 Vgl. K. Hörmann, Lexikon, S. 702. Des Weiteren, so Hörmanns Argumentation, könne man ohne die Erlaubnis zur Verteidigung der ungerechten Gewalt keinen Einhalt gebieten. Man müsse einem Volk die Verteidigung im Falle eines Angriffs zugestehen und zwischen dem Einsetzen von militärischen Mitteln im Falle der Verteidigung und des Angriffs unterscheiden. An dieser Stelle verweist er auf Pius XII., welcher die Völker in der Verpflichtung sah, einem bedrohten Volk zu helfen. Gegen die Argumente, welche für die Einhaltung des Gebotes der Nächstenliebe gegenüber dem Feind plädieren, verweist Hörmann auf die Rangordnung der Dringlichkeit des Liebesgebotes und auf den Umstand der Pflichtenkollision. So sei für einen Staat die oberste Priorität der Schutz seines Volkes und nicht die Schonung des Angreifers. Dies sei immer dann geboten, wenn man zum Schutz anderer verpflichtet sei, im Gegensatz zu einem persönlichem Angriff, bei welchem jeder frei entscheiden könne, ob er einen Angreifer durch ein Nichtwehren „moralisch entwaffnet“. Vgl. K. Hörmann, Lexikon, S. 711-712.

14 K. Hörmann, Friede und moderner Krieg, S. 41. 
Spes“ festgehalten wurde. ${ }^{15}$ „Mit gutem Grund kann man daher sagen: Wer heute einen K. auslöst, handelt unverantwortl. Der Angriffs-K. ist in unserer Zeit auf jeden Fall abzulehnen. “16 Trotzdem findet sich in Hörmanns Werken kein Plädieren für ein allgemeines Kriegsverbot von Seiten der Katholischen Kirche ${ }^{17}$, was auch der theologischen Linie seiner Zeit entsprach. Dennoch gab es sowohl auf Seiten katholischer TheologInnen als auch von der Gesellschaft viel Kritik an diesen Anschauungen. Hörmanns Lehren scheinen bereits in den 60er Jahren nicht nur auf positive Resonanz gestoßen zu sein. In einer Spiegelausgabe aus dem Jahr 1969 - einem Spezial über den Vietnamkrieg -, findet sich eine harsche Kritik an der katholischen Lehre des „gerechten Krieges“ und insbesondere an Hörmanns Lexikonartikel. Der unbekannte Autor stützt sich vor allem auf den „Bensberger Kreis“, welcher in den 60er Jahren ein Memorandum herausgab, das sich gegen die traditionelle kirchliche Kriegslehre stellte und ausdrücklich Missbilligung an der katholischen Haltung zum Vietnamkrieg übte. „Katholische Moraltheologen lehnen seit Jahrhunderten ein striktes Nein zum Krieg ab und berufen sich auf Kirchenväter, Päpste und sogar auf Gott selbst“"18 , so die Kritik. Der Autor zweifelt sowohl an der Existenz tatsächlicher Gründe für einen ,gerechten Krieg" als auch an der Möglichkeit eines echten Präventivkrieges. ${ }^{19}$ Auch

15 Vgl. K. Hörmann, Friede und moderner Krieg, S. 41; Überlegungen über den Krieg, S. 7-8; GS 79-82.

16 K. Hörmann, Lexikon, S. 711.

17 Vgl. K. Hörmann, Lexikon, S. 718-720. „Hie und da wurde vorwurfsvoll das Verlangen geäußert, der Papst solle ganz einfach jeden Krieg verbieten. Abgesehen davon, daß der Erfolg eines solchen Verbotes sehr problematisch wäre, kann man ein allgemeines Kriegsverbot nach unseren Darlegungen vom Papst nicht erwarten. Was man von ihm erwarten darf, ist die Unterweisung darüber, welcher Krieg erlaubt und welcher unerlaubt ist, ferner Mahnungen zum Frieden und zur Friedensgesinnung." K. Hörmann, Friede und moderner Krieg, S. 73. Die Kirche verändert ihre Position dahingehend, den Krieg aus ethischen Gründen zu verwerfen und Friedensvorkämpfer zu sein, wie es Pius XII. und Johannes XXIII., gemacht hätten. Friede könne jedoch nur in einem Ordnungsgefüge verwirklicht werden, welches in Wahrheit gründet, nach den Richtlinien der Gerechtigkeit erbaut wird, von Liebe erfüllt und in Freiheit getragen wird. Vgl. K. Hörmann, Friede und moderner Krieg, S. 73-75.

18 „Schmiedet Scharen“, in: Spiegel Online, online abrufbar unter: http://www.spiegel.de/spiegel/print/d-45741194.html [01.07.2017]. Der Autor kritisiert darüber hinaus, dass die katholische Kirche jegliche Form der Revolution ablehnt.

19 Dieser Meinung schließt sich auch Hörmann an. So verweist er darauf, dass Hitler seine Angriffskriege als Präventivkriege getarnt hat, ebenso sei es im Koreakrieg geschehen. Unechte Präventivkriege seien daher wie Angriffskriege zu behandeln. Man dürfe zwar gegen einen unmittelbar bevorstehenden Angriff Notwehr üben, durch moderne Waffen habe sich jedoch der Begriff der Unmittelbarkeit verändert, zumindest was die Räumlichkeit betreffe. Erst wenn eine Regierung höchste Gewissheit über einen Angriff habe, dürfe sie einem Angriff zuvorkommen, im Idealfall mit vorhergehenden 
Birgit Rath

das Andenken eines Gebrauchs von Atomwaffen, welche Hörmann in seinem Artikel $^{20}$ unter bestimmten Umständen billigt, stößt auf strikte Ablehnung. „So militant geben sich Papst und katholische Bischöfe heutzutage nicht mehr.“21

An dieser Stelle sollte jedoch hinzugefügt werden, dass Hörmann für den Einsatz von Atomwaffen Bedingungen aufstellt, welche ihre Nutzung sehr stark einschränken. ${ }^{22}$ Diese Position vertraten auch einige der führenden Moraltheologen gegen Ende der 50er Jahre im Zuge der Atomkriegsdebatte. ${ }^{23}$ Darüber hinaus betont Hörmann die Verwerflichkeit eines Einsatzes dieser Waffen gegenüber Zivilisten. Auch sei ihre Anwendung, etwa auf militärische Ziele, auf Grund der ungeheuren Schädigungen gegenüber Mensch und Umwelt, ebenfalls fraglich, da angezweifelt werden könne, ob die Schädigungen tatsächlich eingrenzbar seien, wie es manchmal von der Wissenschaft dargestellt werde. Des Weiteren stellt Hörmann in Frage, ob es militärische Ziele geben könne, die wichtig genug seien, um solch großen Schaden bei der Zivilbevölkerung in Kauf

Warnungen und Ultimaten. Doch diesbezüglich hegt Hörmann viele Bedenken. So sei der Erfolg nicht sicher, man gäbe dem Gegner den Vorteil in die Hand, der Angegriffene zu sein, und es stellt sich die Frage, wie es überhaupt gelingen könne, vollkomme Gewissheit zu erlangen, da Beschlüsse auch kurzfristig geändert werden können. Vgl. K. Hörmann, Friede und moderner Krieg, S. 71-72. So schreibt Hörmann in seiner Conclusio: „Man wird daher nicht umhin können, gegen einen heutigen Präventivkrieg stärkste Bedenken zu hegen.“ K. Hörmann, Friede und moderner Krieg, S. 72.

20 Vgl. K. Hörmann, Friede und moderner Krieg, S. 59.

21 http://www.spiegel.de/spiegel/print/d-45741194.html [01.07.2017].

22 So dürfen Atomwaffen nur gegen militärische Ziele angewandt werden und auch nur dann, wenn keine Zivilpersonen geschädigt werden und diese Handlung keinen internationalen Vereinbarungen entgegensteht. Verboten sei hingegen ein Anwenden gegen Zivilpersonen, um ein schnelleres Kriegsende herbeizuführen, da es sich um eine direkte Tötung von Nicht-Kämpfern handelt. Nicht verwendet werden dürfen hingegen Waffen, deren Kontrollierbarkeit nicht gewährleistet sei. Vgl. K. Hörmann, Lexikon, S. $718-720$.

23 So verkündeten sieben führende Moraltheologen und Sozialethiker (u.a. Alfons Auer, Joseph Höffner und Nikolaus Monzel) 1958 in einer Meldung, dass die Anwendung von Atomwaffen sich nach dem Urteil Sachkundiger nicht der völligen Kontrollierbarkeit entzieht und dass deren Gebrauch somit nicht in jedem Fall als Sünde angesehen werden kann. Dieser Meinung vertraten jedoch nicht alle TheologInnen und PhilosophInnen. An dieser Stelle bricht Hörmann auch mit der Lehre Pius XII., welcher die Kontrollierbarkeit der Atomwaffen nicht mehr als gegeben betrachtete und deren Verwendung somit sittlich verurteilt. Für Pius XII. lag das zentrale Problem in diesem Diskurs jedoch nicht im militärischen, sondern im politischen Gebrauch von Atomwaffen als Machtinstrument. Vgl. Nagel, Ernst-Josef / Oberhem, Harald, Dem Frieden verpflichtet, S. 25, S. 33. Zur genaueren Nachlese: Fleischmann, Rudolf / Spaemann, Robert u.a. (Hrsg.), Atomare Kampfmittel und christliche Ethik. Diskussionsbeiträge deutscher Katholiken, Kösel, München, 1960. 
zu nehmen. ${ }^{24}$ Ungewiss bleibt für ihn auch, ob sich Umstände vorstellen lassen, welche die Anwendung von Kernwaffen rechtfertigen. Keinesfalls dürfe man eine solche gutheißen oder empfehlen. ${ }^{25}$

Allgemein kann nach eingehender Betrachtung seiner Artikel der Vorwurf, er würde sich nicht eindeutig gegen den Einsatz von atomaren Waffen stellen, nicht widerlegt werden, da er sich nie dezidiert dagegen ausspricht. Doch die diversen Einschränkungen, die er für die Benutzung aufstellt, legen die Vermutung nahe, dass diese Bedingungen niemals erfüllt werden können, was einem Verbot gleichkommt.

Die Frage, die sich stellt, ist, warum sich Hörmann immer wieder dezidiert gegen Verbote stellt. Dies lässt sich vermutlich aus seiner Ablehnung einer „Verabsolutierungsethik“ erklären, welche unter anderem auf eine theologische Entwicklung des 20. Jahrhunderts zurückzuführen ist. So hat man in der katholischen Theologie versucht, dem Menschen, seiner Vernunft und seinen Gewissensentscheidungen ${ }^{26}$ mehr eigene Verantwortung $\mathrm{zu}$ übertragen und Moraltheologie nicht bloß auf Ver- und Gebote zu beschränken.

Wie in vielen anderen Belangen versucht Hörmann, eine Verabsolutierungsethik zu vermeiden. Absolute Antworten könne man nicht von der Kirche verlangen, da diese Antworten niemals den gegebenen Umständen vollkommen entsprechen könnten.

24 Vgl. K. Hörmann, Friede und moderner Krieg, S. 58.

25 Vgl. K. Hörmann, Friede und moderner Krieg, S. 60.

26 Dies lässt sich auch an Hörmanns Haltung gegenüber der Wissenschaft erkennen. Ob Wissenschaftler an der Entwicklung von atomaren Waffen mithelfen, sei ein Gewissensentscheid. Er fügt jedoch, im Sinne Pius XII. hinzu, dass er der Ansicht sei, nicht jede Forschung gehöre in die Hand von jedermann. Wenn etwa ein schlechter Gebrauch gefürchtet werden muss, dürfe sie nicht weitergegeben werden, da man sich sonst am Bösen mitschuldig macht. Wissenschaft sei dann nicht gut, wenn sie die Absicht habe, anderen Schaden zuzufügen. Niemals dürfe eine Ungerechtigkeit unterstützt werden, auch nicht die der eigenen Nation, dies gelte für Wissenschaftler, aber auch für Ärzte etc. Als ein weiteres Beispiel sei der Militärdienst zu nennen. Hörmann nennt unterschiedliche Bedenken, die gegen den Militärdienst vorgebracht werden, etwa das Ablehnen von Gewaltanwendung, weiters den heutigen Krieg mit modernen Waffen oder einen Krieg, dem Ungerechtigkeit zugeschrieben wird. Auch kann der Militärdienst als Vorbereitung auf einen ungerechten Krieg gesehen werden. Über Menschen, die gegen jede Gewaltanwendung seien, sagt Hörmann, dass diese objektiv irren. Bei den anderen Bedenken sei die Kirche nicht in erster Linie für die Beantwortung der Fragen zuständig. Man könne sich an Experten oder an die Führung des Staates halten, wenn dieser ein rechtmäßiger ist, doch auch hier bleibt Hörmann sehr vage. Über eine „eigene Stellungnahme nach bestem Wissen und Gewissen“ könne man, sei es als Last oder als Vorrecht empfunden, nicht herumkommen. Vgl. K. Hörmann, Friede und moderner Krieg, S. 61-69. 
Birgit Rath

„Antworten, die in die Form des Wenn gekleidet sind, mögen als unbefriedigend erscheinen. Die Menschen wollen meistens eindeutige Auskünfte. Pius XII. hat während seines Pontifikates um genaue Antworten gerungen. Auch die Väter des Zweiten Vatikanischen Konzils haben sich darum abgemüht. Aber sind bis ins letzte gehende Antworten überhaupt möglich? Karl Rahner warnt einmal vor einer solchen Erwartung: Es sei nicht so, daß die Kirche ohnehin die genauen Anweisungen gebe und daß die Übel in der Welt nur aus der Nichtbefolgung dieser Anweisungen entspringen; es sei aber auch nicht so, daß die kirchlichen Stellen genaue Antworten geben könnten, jedoch aus Trägheit oder anderen Gründen es nicht tun. Vielmehr handle es sich um Fragen, zu denen man für den Einzelfall gültige Erklärungen vom kirchlichen Lehramt zu Unrecht erwarte, weil die konkrete Richtigkeit des Verhaltens sehr von den gegebenen Tatsachen abhänge, die von den beteiligten Menschen selbst gewissenhaft zu beurteilen seien. ${ }^{\text {27 }}$

An dieser Stelle fragt Hörmann berechtigterweise an, ob ethische Diskussionen über den modernen Krieg überhaupt sinnvoll seien. Dies kann allerdings bejaht werden, weil solcherlei Argumentationen, auch wenn in ihnen keine vorgefertigten Antworten auf konkrete Probleme gefunden werden, dennoch Hilfestellungen für den Einzelnen bieten, zu eigenverantwortlichen Entscheidungen zu kommen und sich dementsprechend für den Frieden einzusetzen. ${ }^{28}$

„Die Kirche und die Moraltheologen können nicht konkrete Lösungen vorfabrizieren, ja sie dürfen es nicht einmal tun, weil sie damit in unzulässiger Weise dem einzelnen seine eigene Verantwortung abnehmen würden. Wenn die konkreten Lösungen aber letztlich vom beteiligten Einzelmenschen zu finden sind, wird man immer darauf gefaßt sein müssen, daß auch gewissenhafte Menschen zu verschiedenen Ergebnissen kommen, weniger, weil sie im Grundsätzlichen nicht übereinstimmen, als vielmehr deshalb, weil sie die konkrete Lage und ihre Möglichkeiten verschieden beurteilen. “29

So steht fest, auch wenn sich in Hörmanns Lehre kein generelles Kriegsverbot findet und etwa die Anwendung von Atombomben in seinem Lexikonartikel unter bestimmten Bedingungen gebilligt wird, dass aus seiner Inaugurationsre$\mathrm{de}^{30}$ klar seine Ablehnung gegenüber diesen Kriegsmitteln herausgelesen werden kann. Die Autorin hegt die Vermutung, dass Hörmann durch seine eigenen Erfahrungen, die er im Zweiten Weltkrieg und unter dem Unrechtsregime des Nationalsozialismus gemacht hat, zu der Einsicht gekommen ist, dass ein Eingreifen gegen solche Mächte als gerechtfertigte Verteidigung möglich sein muss. Darüber hinaus dürfe nach Hörmann eine totale Gewaltanwendung nicht verboten werden, um auch in Zukunft eine Abschreckung bzw. eine funktionierende Abwehr gegen solche Regierungen zu haben. Denn solange keine gesellschaft-

27 K. Hörmann, Überlegungen über den Krieg, S. 10.

28 Vgl. K. Hörmann, Überlegungen über den Krieg, S. 11.

29 K. Hörmann, Überlegungen über den Krieg, S. 10-11.

30 Vgl. K. Hörmann, Überlegungen über den Krieg, S. 12. 
lichen Strukturen geschaffen werden, in denen solch kriegerische Züge nicht mehr von Nöten sind, müsse den Regierungen das Recht auf Verteidigung zugestanden werden. ${ }^{31}$

\section{Der Weg zum Frieden}

Offen bleibt, wie solche friedlichen Ordnungsstrukturen geschaffen werden können, in denen man sich für ein Kriegsverbot aussprechen kann. Hörmann sieht die Lösung für die Frage nach der Gestaltung dieser Strukturen nicht in der Bekämpfung der Kriegssymptome und in Verboten, sondern in der „Behebung der verursachenden Übel“‘32. Selbst ein Verteidigungskrieg sei nur Anzeichen eines zuvor begangenen Unrechts und allein deswegen bedauerlich. Hierbei stützt er sich auf Pius XII., der diese Problematik in seinen zahlreichen Gelegenheitsaussagen ${ }^{33}$ eingehend behandelt. Außerdem verweist er auf den protestantischen Theologen Karl Barth ${ }^{34}$. Dieser habe Pazifisten und Militaristen vorgeworfen, dass weder ein „Abrüsten noch ein Aufrüsten cura prior sein dürfe“ ${ }^{\text {(35. }}$. Die einzige Lösung sei das Herstellen einer sinnvollen und gerechten Lebensordnung für alle.

„Wenn eine Ordnung der Gerechtigkeit und der Liebe, wie sie Christus als Ziel hat, verwirklicht ist, kann der Krieg kein Problem sein, weil er jede Funktion verloren hat und überflüssig geworden ist. “36

31 „Auch heute behält der radikale Pazifismus mit seiner absoluten Kriegsgegnerschaft nicht recht. Auch heute ist jene Gewaltanwendung nicht zu verwerfen, die den Charakter der Notwehr eines zu Unrecht angegriffenen Volkes hat oder, nach Begründung einer Staatengemeinschaft mit entsprechender Sanktionsgewalt, eine Polizeiaktion dieser Gemeinschaft gegen ein rechtsbrecherisches Mitglied darstellt (Verteidigungsund Sanktionskrieg). Die apodiktische Verurteilung jeden Krieges kann vor dem kritischen Verstand und vor dem Rechtsgefühl nicht bestehen.“ K. Hörmann, Friede und moderner Krieg, S. 49. Dies bedeutet für ihn jedoch letzten Endes nicht, dass jeder Verteidigungskrieg erlaubt ist, denn auch dieser muss die Voraussetzungen eines gerechten Krieges erfüllen.

32 K. Hörmann, Lexikon, S. 702.

33 Vgl. Utz, Arthur-Fridolin / Groner, Joseph-Fulko (Hrsg.): Aufbau und Entfaltung des gesellschaftlichen Lebens. (Soziale Summe Pius XII. Bd. 3), Paulusverlag, Freiburg, 1961, S. 3854, UG 6405.

34 Siehe auch, Barth, Karl, Kirchliche Dogmatik. Die Lehre von der Schöpfung, Bd. III, Zollikon, Zürich, ${ }^{2} 1957$.

35 K. Hörmann, Überlegungen über den Krieg, S. 6.

36 K. Hörmann, Überlegungen über den Krieg, S. 6. 
Birgit Rath

So betont Hörmann trotz aller Verteidigung der früheren Lehren und dem Festhalten an einem Recht auf Verteidigung, dass sich die katholischen Theologen einig seien, dass kein Grund schwerwiegend genug sei, heute einen Krieg heraufzubeschwören. ${ }^{37}$

Aufgabe der Kirche sei es, dem Friedenswirken zu dienen und die Menschen zu einer Friedensordnung zu drängen. Bei der Definition der zu erfüllenden gesellschaftlichen Strukturen, bleibt Hörmann allerdings vage. Es solle eine Friedensordnung geschaffen werden, welche einem „Werk der Gerechtigkeit“ entspräche, wie sie auch in „Gaudium et Spes“ 78 benannt wird. Das Zweite Vatikanische Konzil habe erkannt, dass echter Friede nicht erst mit dem „Nichtkriegführen" gelingen kann, sondern dem Charakter nach etwas Dynamisches ist, das nur durch die ständige Suche nach Gerechtigkeit und Liebe gesichert werden kann. $^{38}$

Das friedliche Zusammenleben ist allerdings an einige wichtige Grundlagen gebunden. Zunächst sei es essentiell, die Menschenrechte als grundlegend anzuerkennen. Darüber hinaus müsse man den „,aufstrebenden Völkern“ Entwicklungshilfe leisten, wie es etwa in der Enzyklika Pauls VI. „Populorum Progressio“ 43-86 und in „Gaudium et Spes“ 81, 85-97 festgehalten wurde. Als weiteres Mittel zur Herstellung friedlicher Strukturen, nennt Hörmann in Bezugnahme auf „Gaudium et Spes“ 90 die Schaffung eines Organs der Gesamtkirche, dessen Aufgabe es sein solle, die Katholiken anzuregen, den Aufstieg der notleidenden Gebiete und die soziale Gerechtigkeit zu fördern. Dieses Organ sieht Hörmann in der 1967 von Paul VI. errichteten Kommission „Iustitia et Pax“ verwirklicht. ${ }^{39}$ Sozialer Fortschritt, Ordnung, Sicherheit und Ruhe der einzelnen Staaten seien weiterhin zur Friedenssicherung notwendig, wie dies auch von Johannes XXIII. und Paul VI. betont wurde.

Hörmann sieht als Ziel des Friedenswirkens der Kirche das Ausschalten des Krieges. Dies sei bereits auf dem Ersten Vatikanischen Konzil angeklungen und habe sich in Friedensgesuchen unter den Päpsten Pius IX., Benedikt XV. und Pius XII. fortgesetzt. Benedikt XV. etwa urteilte in eindeutiger Weise über den Ersten Weltkrieg, indem er ihn wörtlich als ,Suicidio dell'Europa civile“ ${ }^{\text {“40 }}$ bezeichnete. $^{41}$

Zur Friedenssicherung wiederum benötige man eine Friedensorganisation, deren hauptsächliches Ziel es sein sollte, internationale Zusammenarbeit zu

37 Vgl. K. Hörmann, Überlegungen über den Krieg, S. 7.

38 Vgl. Hörmann, Karl, Erziehung zum Frieden durch Versöhnung, in: Sqicciarini, Donato (Hrsg.), Die Weltfriedensbotschaften Papst Paul VI., Duncker \& Humblot, Berlin, 1979, S. 65-70, hier: S. 66.

39 Vgl. Hörmann, Karl, Lexikon der christlichen Moral, Tyrolia, Innsbruck / Wien / München, 1976, S. 936-937.

40 Acta Apostolicae Sedis 8 (4.3.1916), S. 59.

41 Vgl. K. Hörmann, Friede und moderner Krieg, S. 10-11; Erziehung zum Frieden durch Versöhnung, S. 67. 
fördern, um so Kriege zu verhindern. Hintergrund für diese Friedensorganisation bietet der Gedanke einer Völkergemeinschaft. Dies könne etwa in den Bemühungen der Vereinten Nationen verwirklicht gesehen werden. ${ }^{42}$ Weiters bräuchte es eine Kontrolle von ABC-Waffen etwa in Form von Rüstungsabkommen und Rüstungseinschränkungen, für deren Einsatz sich bereits Pius XII. eingesetzt habe und deren Einhaltung ebenfalls von den Vereinten Nationen überwacht werden solle. Ein weiterer Punkt für das Erlangen des Friedens seien die Erfüllung der Forderungen der Minderheiten, eine gerechte Verteilung von Gütern, eine Erleichterung der Ein- und Auswanderungspolitik oder etwa der freie Zugang zu Rohstoffen. Als Grundlage für das Erfüllen dieser Bedingungen sieht Pius XII., dass sich die Regierenden nach dem göttlichen Recht und dem Gesetz der Sittlichkeit ausrichten. ${ }^{43}$

Darüber hinaus solle es eine Erziehung zur Friedensgesinnung geben. Nach Hörmann sei etwa der Völkerbund daran gescheitert, dass es den Völkern und Lenkern an einer echten Friedensgesinnung fehlte. Kleine Staaten seien zur Einhaltung der Grundsätze gezwungen worden, die großen jedoch hätten sich darüber hinweggesetzt. Die Vereinten Nationen könnten so etwas nur verhindern, wenn ihre Mitglieder einen Teil ihrer Souveränität an diese übertrügen. Dieses Opfer müssten Staaten mit einer rechten Friedensgesinnung und einem echten Pazifismus bereit sein zu bringen. Dabei ginge es darum, die Annäherung der Völker zu unterstützen. Eine Arbeit, die nicht erst bei drohendem Krieg getan werden müsse, sondern auch in Zeiten des Friedens. Kriegsverhütende Friedensarbeit müsse in ständigem Bemühen auf weite Sicht bestehen. Der Friedenswille müsse gegenüber dem Kriegswillen siegen, damit nicht jede Kriegsursache auch zu einem solchen führe. Die Öffentlichkeit solle in Richtung eines friedlichen Zusammenlebens beeinflusst werden, einerseits durch das Herstellen von Kontakten, andererseits im Wecken eines Weltbürgerbewusstseins und dem kritischen Hinterfragen von Propagandalügen. ${ }^{44}$ Die Kirche müsse in der Menschheitsfamilie eine Einheit in Gesinnung, im Herzen, im Glauben und in der Liebe wecken, wie es etwa mit der Pax-Christi Bewegung versucht werde. ${ }^{45}$

42 Vgl. K. Hörmann, Friede und moderner Krieg, S. 12-15; Unterwegs zum Frieden, S. 364-365.

43 Vgl. K. Hörmann, Friede und moderner Krieg, S. 19.

44 Vgl. K. Hörmann, Friede und moderner Krieg, S. 21-22.

45 Vgl. K. Hörmann, Unterwegs zum Frieden, S. 366. 
Birgit Rath

\section{Ausblick}

Mag die Theorie eines „gerechten Krieges“ auch überholt scheinen, findet sie doch bis heute Anwendung im moraltheologischen Diskurs ${ }^{46}$ und es gibt Anzeichen einer Wiederkehr der bellum-iustum-Lehre in umgestalteter Form. ${ }^{47}$ Eberhard Schockenhoff weist sogar darauf hin, dass eine wachsende Zahl von Autoren empfiehlt, diese nicht vorschnell zu verabschieden, sondern viel eher von „späteren Überlagerungen und Kontaminierungen zu befreien, zugleich aber ihre moralische Kriteriologie beizubehalten und diese in eine umfassende Theorie internationaler Beziehungen oder Weltrechtsordnung einzubauen “48. Sie könne demnach als Ergänzung der aktuellen Lehre vom gerechten Frieden dienen, vor allem in Bezug auf die Frage nach dem Einsatz von militärischer Gewalt. ${ }^{49}$

Doch sollten Begriffe wie bellum iustum heute überhaupt noch Anwendung innerhalb der Theologie finden? Müsste man sich nicht in jeder Hinsicht gegen die Möglichkeit eines „gerechten Krieges“ aussprechen, da die Bedingungen ohnehin nicht erfüllt werden können und er immer Ungerechtigkeiten mit sich brächte? Wie wäre dann mit der Tatsache umzugehen, dass die gerechte Gesell-

46 Eberhard Schockenhoff etwa schreibt in seiner Ethik des Lebens, dass selbst in einem „gerechten Krieg“ das Töten keineswegs erlaubt sei. Als „gerecht“ sei er demgemäß anzusehen, wenn er der Verteidigung des eigenen Landes oder der Verhinderung einer systematischen Menschenrechtsverletzung diene. Vgl. Schockenhoff, Eberhard, Ethik des Lebens. Grundlagen und neue Herausforderungen, Herder, Freiburg / Basel / Wien, 2009, S. 269. Diese Argumentation folgt derselben Logik wie Hörmanns Lexikonartikel. Auch bei Helmut Weber findet sich eine ähnliche Argumentation im Kapitel über Krieg. Vgl. Weber, Helmut, Spezielle Moraltheologie. Grundfragen des christlichen Lebens, Styria, Graz / Wien / Köln, 1999, S. 234-261.

47 Zur genaueren Nachlese: Vgl. Schockenhoff, Eberhard, Welche Impulse kann Theologie der Friedensethik geben?, in: Bock, Veronika / Frühbauer, Johannes J. / Küppers, Arnd / Sturm, Cornelius (Hrsg.), Christliche Friedensethik vor den Herausforderungen des 21. Jahrhunderts, Aschendorff, Münster, 2015, S. 47-71, hier: S. 69.

48 E. Schockenhoff, Welche Impulse kann Theologie der Friedensethik geben?, S. 69.

49 Vgl. E. Schockenhoff, Welche Impulse kann Theologie der Friedensethik geben?, S. 69. Schockenhoff kritisiert in seinem Artikel etwa, dass die bellum-iustum-Lehre oftmals fälschlicherweise als Legitimationsgrund für Kriege interpretiert wurde, ihre ursprüngliche Intention sei allerdings eine kriegskritische gewesen, da die meisten Kriege der Weltgeschichte nach ihren Kriterien als illegitim zu betrachten seien. Somit beruhe diese Lehre auf derselben Grundannahme wie die später etablierte Lehre vom gerechten Frieden, deren beider Ziel, der Friede sei. Als weiteren Grund für eine gewisse Wertschätzung nennt er die Kriterien, welche die bellum-iustum-Lehre in Hinsicht auf die Gewaltanwendung zur Verhinderung von Unrecht und zum Schutz von Rechtlosen aufstellt. Neuere Ansätze würden beispielsweise keinerlei Rechtfertigungsgründe für humanitäre Interventionen bieten. Vgl. E. Schockenhoff, Welche Impulse kann Theologie der Friedensethik geben?, S. 65-69. 
schaftsordnung noch nicht geschaffen wurde und es immer noch Kriege gibt? Das Zweite Vatikanische Konzil brachte in Bezug auf die Problematik des Krieges keine umfassenden Antworten. Viel eher versuchte man, sich auf die Schaffung einer gerechten Ordnung und einer Friedensgesinnung zu konzentrieren. Weiters wurden atomare Waffen zunächst noch als geeignete Mittel zur Abschreckung gesehen, erst 1983 sprach sich die US-amerikanische Bischofskonferenz für ein Verbot von Atomwaffen aus. Schließlich wurden sie von Papst Johannes Paul II. 1997 vor dem Komitee der Vereinten Nationen verdammt. ${ }^{50}$

Hörmanns Schriften sind durchgehend von diesem Zwiespalt geprägt. Einerseits zeigen sie das Festhalten an der traditionellen Lehre vom gerechten Krieg und an dem Recht auf Verteidigung auf, andererseits werden bereits Versuche einer neuen Friedensethik sichtbar. Ähnlich wie Pius XII. schreibt und denkt Hörmann „zwischen den Zeiten“ und steht in gewisser Weise paradigmatisch für ein Dilemma seiner Zeit. So war seine Lehre von der Verpflichtung der Verteidigung von Gerechtigkeit und gleichzeitig von der Sehnsucht nach Frieden geprägt.

Die aktuellen gesellschaftlichen Verhältnisse bedürfen zeitgerechter Antworten. Als Hörmann seine Schriften verfasste, war die Frage nach den Atomwaffen ein aktueller Bezugspunkt und er hat versucht, diese aus Sicht seiner Zeit zu beantworten. Heute gibt es neuere Erkenntnisse und demgemäß eine strikte Ablehnung gegenüber Atomwaffen. Dennoch müssen wir uns weiterhin mit dieser Problematik auseinandersetzen, ebenso mit der Frage, welche ethischen Kriterien bei militärischen Gewaltanwendungen in bestimmten Situationen angewandt werden können. Diese Fragen wurden noch nicht in eindeutiger Weise geklärt und man sieht sich nach wie vor mit ähnlichen Überlegungen, wie sie Mitte des letzten Jahrhunderts angestellt wurden, konfrontiert. In diesem Sinne können Hörmanns Schriften und vor allem seine Forderungen nach einer zeitgemäßen Theologie auch gegenwärtig hilfreich sein, um ein umfassenderes Bild in einem sehr komplexen Diskurs zu bekommen.

\section{Literaturverzeichnis}

Barth, Karl: Kirchliche Dogmatik. Die Lehre von der Schöpfung, Bd. III, Zürich, ${ }^{2} 1957$. Beestermöller, Gerhard: „Krieg“, in: Hunold, Gerfried W. / Sautermeister, Jochen (Hrsg.): Lexikon der christlichen Ethik Bd. 1, Freiburg / Basel / Wien, ${ }^{3} 2003$, S. 1015.

Fleischmann, Rudolf / Spaemann, Robert u.a. (Hrsg.): Atomare Kampfmittel und christliche Ethik. Diskussionsbeiträge deutscher Katholiken, München, 1960.

Hörmann, Karl: „Sittliche Probleme des modernen Krieges“, in: Religion - Wissenschaft - Kultur 8 (1957), S. 221-226.

Hörmann, Karl: Friede und moderner Krieg im Urteil der Kirche, Wien, 1964. Hörmann, Karl: Eid, Befehl, Widerstand, Innsbruck / Wien / München, 1964.

50 Vgl. H. Weber, Spezielle Moraltheologie, S. 254-255. 
Birgit Rath

Hörmann, Karl: Der Wert sittlicher Überlegungen über den Krieg. Inaugurationsrede gehalten am 20. Oktober 1966, Wien, 1966.

Hörmann, Karl: Lexikon der christlichen Moral, Innsbruck / Wien / München, 1969.

Hörmann, Karl: Wehrdienst - Kriegsdienst - Verweigerung - gerechter Krieg?, Augsburg, 1971.

Hörmann, Karl: „Der ,gerechte Krieg“ im christlichen Denken“, in: Weiler, Rudolf / Zsifkovits, Valentin (Hrsg.): Unterwegs zum Frieden, Freiburg / Basel / Wien, 1973, S. 335-367.

Hörmann, Karl: Lexikon der christlichen Moral, Innsbruck / Wien / München, 1976.

Hörmann, Karl: „Erziehung zum Frieden durch Versöhnung“, in: Sqicciarini, Donato (Hrsg): Die Weltfriedensbotschaften Papst Paul VI., Berlin, 1979, S. 65-70.

Jensen, Jessica: Krieg um des Friedens willen. Zur Lehre vom gerechten Krieg, BadenBaden, 2015.

Nagel, Ernst-Josef / Oberhem, Harald: Dem Frieden verpflichtet. Konzeptionen und Entwicklungen der katholischen Friedensethik seit dem Zweiten Weltkrieg, München / Mainz, 1982.

Schockenhoff, Eberhard: Ethik des Lebens. Grundlagen und neue Herausforderungen, Freiburg / Basel / Wien, 2009.

Schockenhoff, Eberhard: „Welche Impulse kann Theologie der Friedensethik geben?“, in: Bock, Veronika / Frühbauer, Johannes J. / Küppers, Arnd / Sturm, Cornelius (Hrsg.): Christliche Friedensethik vor den Herausforderungen des 21. Jahrhunderts, Münster, 2015, S. 47-71.

Virt, Günter (Hrsg.): Spiritualität in Moral. Festschrift für Karl Hörmann zum 60. Geburtstag, Wien, 1975.

Virt, Günter: „Karl Hörmann“, in: Bautz, Friedrich Wilhelm / Bautz, Traugott (Hrsg.): Biographisch-Bibliographisches Kirchenlexikon Bd.27, Ergänzungen XIV, Nordhausen, 2007, S. 674.

Weber, Helmut: Spezielle Moraltheologie. Grundfragen des christlichen Lebens, Graz I Wien / Köln, 1999.

Utz, Arthur-Fridolin / Groner, Joseph-Fulko (Hrsg.): Aufbau und Entfaltung des gesellschaftlichen Lebens. (Soziale Summe Pius XII. Bd. 3), Paulusverlag, Freiburg, 1961, S. 3854.

\section{Internetquellen}

„Schmiedet Scharen“, in: Spiegel Online, online abrufbar unter: http://www.spiegel.de/ spiegel/print/d-45741194.html [01.07.2017].

Gaudium et Spes. Pastorale Konstitution über die Kirche in der Welt von Heute, online abrufbar unter: http://www.vatican.va/archive/hist_councils/ii_vatican_council/docu ments/vat-ii_const_19651207_gaudium-et-spes_ge.html [01.08.2017].

Kathpedia: Karl Hörmann, online abrufbar unter: http://www.kathpedia.com/index. php?title=Karl_H\% C3\% B6rmann [09.02.2017].

Populorum Progressio, online abrufbar unter: http://w2.vatican.va/content/paul-vi/de/ encyclicals/documents/hf_p-vi_enc_26031967_populorum.html [07.02.2018]. 
Birgit Rath, Department of Systematic Theology and Ethics, Faculty of Catholic Theology, University of Vienna, Schenkenstraße 8-10, 1010 Wien, Austria, e-mail: birgit. rath@univie.ac.at

Citation: Rath, Birgit: „Bellum Iustum - Eine moraltheologische Auseinandersetzung bei Karl Hörmann“, in: Kallhoff, Angela / Schulte-Umberg, Thomas (eds.): Moralities of Warfare and Religion (J-RaT 2018 / 1) pp. 207-223.

Datum der Publikation: 16.07.2018 